\title{
Treatment patterns and overall survival among patients with unresectable, stage III non-small-cell lung cancer
}

\author{
Priyanka Bobbili1, Kellie Ryan*,2, Mei S Duh1, Akanksha Dua1(iD), Ancilla W Fernandes², \\ Melissa Pavilack² \& Jorge E Gomez ${ }^{3}$ \\ ${ }^{1}$ Analysis Group, Inc., Boston, MA 02199, USA \\ ${ }^{2}$ US Medical Affairs, AstraZeneca, Gaithersburg, MD 20878, USA \\ ${ }^{3}$ Icahn School of Medicine at Mt Sinai, New York, NY 10029, USA \\ *Author for correspondence: Tel.: +1 301398 5355; Kellie.Ryan@astrazeneca.com
}

\begin{abstract}
Aim: To analyze treatment patterns and overall survival (OS) across time (2009-2014) among patients with unresected, stage III non-small-cell lung cancer (NSCLC). Patients \& methods: Stage III NSCLC patients aged $\geq 65$ years who initiated therapy were identified using SEER-Medicare data. Results: Among 4564 patients, $84 \%$ received chemotherapy (with or without radiotherapy), and $59 \%$ received chemoradiotherapy (CRT). Carboplatin + paclitaxel was the most frequent regimen. Median (interquartile range) OS among chemotherapy patients was 13.2 (6.0-28.9) months, and 14.8 (6.7-33.4) months among CRT patients. Among CRT patients, there was no difference in OS across years of CRT initiation. Conclusion: OS remained static across 2009-2014, indicating stagnancy in clinical outcomes for stage III NSCLC patients and a need for more effective therapeutic options.
\end{abstract}

First draft submitted: 15 May 2019; Accepted for publication: 2 August 2019; Published online:

23 September 2019

Keywords: chemoradiotherapy $\bullet$ chemotherapy $\bullet$ medicare $\bullet$ multi-modality SEER medicare $\bullet$ standard-of-care

Lung cancer is the leading cause of cancer-related deaths in the USA, with an estimated 234,030 new cases and 154,030 deaths expected in 2018 [1]. Non-small-cell lung cancer (NSCLC) is the most common type of lung cancer, accounting for $80-85 \%$ of all newly diagnosed lung cancer cases. Approximately a third of all NSCLC patients present with stage III disease [2-5]. The 5-year survival rate of stage III NSCLC ranges from 13 to $36 \%$, and is further reduced once the cancer has metastasized to other regions of the body [2].

The standard-of-care for patients with unresectable, stage III NSCLC for the last three decades has been platinumbased doublet chemotherapy regimens administered concurrently with radiotherapy (i.e., chemoradiotherapy or CRT), followed by active surveillance [6-8]. Despite curative intent, efficacy remains poor in patients receiving CRT, with the majority of patients progressing and later dying of the disease. A 2010 meta-analysis reported that median progression-free survival among patients treated with CRT is 8 months, and that the 5 -year overall survival (OS) is approximately $15 \%[9]$.

Multiple strategies to improve survival in these patients have been studied in clinical trials. Studies testing induction chemotherapy [10,11], consolidation therapy and radiation dose escalation have failed to produce improvements in survival in randomized Phase III trials [12]. The treatment landscape in patients with unresectable, stage III NSCLC was largely unchanged until the recent approval of durvalumab, a selective, high-affinity, ant-PD-L1 human IgG1 monoclonal antibody [13].

To understand the impact of new treatment options for stage III NSCLC, it is important to examine the existing state of the therapeutic landscape. The current study aimed to assess real-world treatment patterns and clinical outcomes in Medicare patients, aged 65 years or older, with unresectable, stage III NSCLC to fill this gap in knowledge and to define unmet medical needs in these patients.

Future Medicine 


\section{Patients \& methods}

\section{Surveillance, Epidemiology, \& End Results-Medicare database}

This retrospective longitudinal cohort study was conducted using data from the Surveillance, Epidemiology, and End Results (SEER)-Medicare linked database. The SEER-Medicare database links data from the SEER cancer registries to Medicare enrollment and claims files. SEER cancer registries cover approximately $28 \%$ of the US population and collect information for persons newly diagnosed with cancer; information on the first course of treatment is collected in addition to demographic characteristics, month and year of diagnosis, cancer site, stage at diagnosis, histology and date of death, if applicable. For those in SEER who are Medicare eligible, SEER-Medicare data include claims for covered healthcare services, including hospitalizations, outpatient visits, home healthcare and hospice services. Data for 93\% of SEER patients age 65 years or older are successfully linked to Medicare data [14]. At the time of this analysis, the SEER cancer registries contained data on incident cancer cases between 1991 and 2013, and linked Medicare claims data were available through 2014.

This study was determined to be exempt from Institutional Review Board (IRB) review by the New England IRB as the research involved analysis of secondary de-identified data.

\section{Study design \& population}

This study utilized SEER-Medicare data from 1 January 2009 to 31 December 2014. Data were collected for patients diagnosed with NSCLC between 2009 and 2013, identified using the two-digit cancer site recode in SEER (C34.0-C34.9 'Lung and bronchus') and International Classification of Disease for Oncology (ICD-O-3) morphology codes 8012, 8046, 8070, 8071, 8140, 8250, 8480, 8481, 8490 and 8570. Patients had stage III disease at the time of diagnosis, based on the American Joint Committee on Cancer Tumor, Nodes, Metastases Staging System, Sixth edition. Eligible patients were aged 65 years or older at the time of diagnosis, had coverage for both Medicare Part A and Part B, and no health maintenance organization enrollment. The index date was defined as the date of initiation of the first therapeutic regimen and within 90 days of stage III NSCLC diagnosis. Patients were required to have at least 6 months of continuous insurance eligibility prior to the index date to collect baseline information, and at least 1 month of continuous insurance eligibility after the index date. Patients were excluded from the analysis if the reporting source of their lung cancer diagnosis was an autopsy or death certificate, if they had more than one primary tumor, or if they had surgical resection of their tumor. Surgical resection was identified using both surgical history captured in the SEER registries, which noted whether a cancer-directed surgery was performed within 4 months of NSCLC diagnosis date, and surgical procedures identified using Medicare claims.

A subgroup analysis was conducted among patients who initiated CRT as their first therapeutic regimen for unresected, stage III NSCLC. In addition to meeting the earlier stated inclusion and exclusion criteria, these patients had a claim for chemotherapy within 90 days of stage III NSCLC diagnosis and they received radiation as part of the first course of therapy as identified using data from SEER registries. Information from SEER categorized the radiation received into several types - such as beam radiation or radioactive implants - however, these were not differentiated in our analysis. This subgroup of patients treated with CRT was divided into cohorts defined by index year of CRT initiation, in other words, patients who initiated CRT in 2009-2010, 2011-2012 and 2013-2014.

\section{Variables}

Baseline characteristics were evaluated during the 6 months prior to the index date. Patient demographic characteristics collected at diagnosis included age, sex, race, region and urban setting. Clinical data at diagnosis included histology (e.g., adenocarcinoma, squamous cell carcinoma, etc.), grade, laterality and tumor site. Medicare claims data from the baseline period were used to assess location and number of claims with metastatic site diagnostic codes, Charlson Comorbidity Index (CCI) and proportion of patients tested for tumor markers. Predicted performance status (PS) was estimated using a previously validated multivariate logistic regression model, which predicted a 'good' PS (defined as Eastern Cooperative Oncology Group score 0-2 or Karnofsky Performance Scale score 60-100) or 'poor' PS (Eastern Cooperative Oncology Group score 3-5 or Karnofsky Performance Scale score 0-50) per post-2009 recommendations of the American Society of Clinical Oncology [15].

Therapeutic treatment regimens were captured from claims data using Healthcare Common Procedures Coding System codes for specific agents. Therapeutic regimens (i.e., chemotherapy, targeted therapy, bevacizumab or combinations) were defined as all agents with claims observed within 28 days of the index date. Discontinuation of the first therapeutic regimen was identified by the first occurrence of a claim for a new agent not in the regimen, gaps of more than 90 days between claims for successive agents or end of continuous eligibility/data availability for 
the patient, whichever occurred first [16]. Use of maintenance therapy with erlotinib or pemetrexed following the first therapeutic regimen was defined by a new claim for these agents within 28 days of the end of the regimen [7]. The initiation of the second therapeutic regimen was defined by an NSCLC therapy agent that the patient had not previously received, or an agent that the patient previously received but with a gap greater than 90 days between successive claims. Discontinuation of this second regimen was defined using the same criteria as for the first therapeutic regimen. Duration of therapy was calculated as the number of days from the date of the first agent claim to the date of the last agent claim in the regimen prior to discontinuation, plus 28 days to account for the days of supply for the last claim regardless of mode of administration (e.g., oral or infused).

\section{Statistical analyses}

Descriptive analyses assessing patient baseline and clinical characteristics, as well as treatment patterns, were conducted using means, standard deviations (SD) and medians for continuous variables and frequencies and proportions for categorical variables. OS, defined as the duration in months from the index date to the date of death in SEER, was analyzed using the Kaplan-Meier method to determine median OS and the associated interquartile range. Patients were censored at the earliest of end of eligibility, end of data availability and death. In the subgroup analysis among patients with unresected, stage III NSCLC who initiated treatment with CRT, the proportion of patients alive at 1 year following the index date was compared across study cohorts using the log-rank test, as well as a Cox proportional hazards model adjusted for baseline patient characteristics. All statistical analyses were conducted in SAS Enterprise Guide Version 7.1 (SAS Institute, NC, USA).

\section{Results}

\section{Study population \& baseline characteristics}

Between 2009 and 2014, 4564 patients with unresected, stage III NSCLC met the study inclusion criteria (Figure 1). These patients had a mean age at diagnosis of 74.2 years, $83.5 \%$ were white and $45.3 \%$ were female (Table 1 ). The most frequently observed histology was adenocarcinoma (44.4\%), followed by squamous carcinoma (35.2\%). Among these patients, $38.6 \%$ had stage IIIA disease at diagnosis and $61.4 \%$ had stage IIIB disease; $2.0 \%$ of patients had well-differentiated disease while $29.4 \%$ of patients had poorly differentiated disease. Over half of the patients (61\%) had a good predicted PS. The most frequently observed co-morbidities were hypertension (78.7\%), chronic obstructive pulmonary disease (COPD; 72.6\%), dyslipidemia (67.1\%), ischemic heart disease (39.7\%) and diabetes mellitus (32.0\%). A small proportion of patients (4.3\%) had a lung cancer genomic test conducted during the baseline period for known lung cancer-related mutations/rearrangements, such as EGFR mutations, $A L K$ gene rearrangements and $B R A F$ and $K R A S$ mutations.

Among the subgroup of patients who initiated treatment with CRT $(n=2692)$, baseline demographics and clinical characteristics were similar to the overall study population. Their mean age at diagnosis was 73.5 years, 85.3\% were White and $44.7 \%$ were female (Table 1). Most patients treated with CRT were from the southern US (33.5\%). In terms of clinical characteristics, $1.9 \%$ of patients had a well-differentiated tumor while $32.8 \%$ with a poorly differentiated tumor. Unlike the overall study population, $61 \%$ of patients treated with CRT were diagnosed with stage IIIA NSCLC, 39\% were diagnosed with stage IIIB disease and most patients had squamous cell histology (45\%).

CCI scores were similar between the subgroup of patients who received CRT and the overall study population $(5.5 \pm 2.4$ and $5.7 \pm 2.4$, respectively). Predicted PS was also similar (64.9 and $60.5 \%$, respectively) as was the prevalence of selected co-morbidities (Table 1).

\section{Treatment patterns}

Among the population of patients with unresected, stage III NSCLC who initiated a systemic therapy, $83.8 \%$ $(\mathrm{n}=3826)$ received chemotherapy (with or without radiation), $7.2 \%$ received bevacizumab (with or without combination chemotherapy; $\mathrm{n}=327$ ) and $4.8 \%$ received treatment with a targeted therapy agent (with or without combination chemotherapy; $\mathrm{n}=219$ ) as their first therapeutic regimen (Table 2). Among all patients, $4.3 \%$ received maintenance therapy with pemetrexed or erlotinib, of which most received maintenance therapy following a chemotherapy regimen. Among the patients treated with chemotherapy, $70 \%(\mathrm{n}=2692)$ also received radiotherapy (i.e., received CRT). Patients treated with CRT represented 59\% of the overall population of patients with unresected, stage III NSCLC who initiated a systemic therapy. 
Table 1. Baseline characteristics of patients with unresected, stage III non-small-cell lung cancer.

Baseline characteristics

Age at diagnosis, years (mean \pm SD [median])

Female, $\mathrm{n}(\%)$

Race/ethnicity, $n(\%)$ :

- White

- Black

- Asian

- Hispanic

- Other or unknown

Region, $\mathrm{n}(\%)$ :

- South

- West

- Northeast

- Midwest

AJCC stage:

- IIIA

- IIIB

Grade:

- Well differentiated

- Moderately differentiated

- Poorly differentiated

- Undifferentiated

- Unknown

Histology (ICD-O-3), n (\%):

- Squamous cell (8070)

- Adenocarcinoma (8140)

- General NSCLC (8046)

- Other

Characteristics assessed during baseline period:

Number of unique metastatic sites, mean $\mathrm{SD} \pm$ (median)

Charlson Comorbidity Index, mean \pm SD (median)

Comorbidities:

- Hypertension

- COPD

- Dyslipidemia

- Ischemic heart disease

- Diabetes

- Cerebrovascular disease

- Heart failure

- Chronic kidney disease

- No co-morbidities

Lung cancer genomic tests during baseline period:

- Proportion of patients with $\geq 1$ test, $n$ (\%)

- Number of tests performed, mean \pm SD (median)

Predicted performance status, $\mathrm{n}(\%)$ :

- Good

- Poor

AJCC: American Joint Committee on Cancer; COPD: Chronic obstructive pulmonary disease; ICD-O-3: International Classification of Disease for Oncology-3; NSCLC: Non-small-cell lung cancer; SD: Standard deviation.
Stage III NSCLC patients $(n=$ 4564)

Stage III NSCLC patients receiving chemoradiotherapy $(n=2692)$

$74.2 \pm 6.0(74.0)$

$2066(45.3 \%)$

$73.5 \pm 5.5$ (73.0)

$1202(44.7 \%)$

$3811(83.5 \%)$

$2295(85.3 \%)$

$397(8.7 \%)$

$241(9.0 \%)$

$199(4.4 \%)$

$80(3.0 \%)$

$43(0.9 \%)$

$13(0.5 \%)$

$114(2.5 \%)$

$63(2.3 \%)$

$1377(30.2 \%)$

$1634(35.8 \%)$

901 (33.5\%)

$996(21.8 \%)$

837 (31.1\%)

$557(12.2 \%)$

$580(21.5 \%)$

$374(13.9 \%)$

$1763(38.6 \%)$

1628 (60.5\%)

$2801(61.4 \%)$

1064 (39.5\%)

$93(2.0 \%)$

$52(1.9 \%)$

699 (15.3\%)

$452(16.8 \%)$

$1343(29.4 \%)$

$883(32.8 \%)$

58 (1.3\%)

36 (1.3\%)

2371 (52.0\%)

1269 (47.1\%)

$1613(35.3 \%)$

$1212(45.0 \%)$

2028 (44.4\%)

915 (34.0\%)

$565(12.4 \%)$

$344(12.8 \%)$

$358(7.8 \%)$

$221(8.2 \%)$

$1.0 \pm 1.0(1.0)$

$1.0 \pm 0.9(1.0)$

$5.7 \pm 2.4(6.0)$

$5.5 \pm 2.4(6.0)$

3589 (78.7\%)

2088 (77.6\%)

3313 (72.6\%)

2052 (76.2\%)

$3062(67.1 \%)$

1809 (67.2\%)

$1812(39.7 \%)$

$1077(40.0 \%)$

1459 (32.0\%)

$819(30.4 \%)$

$1084(23.8 \%)$

$669(24.9 \%)$

$747(16.4 \%)$

$368(13.7 \%)$

$454(10.0 \%)$

$238(8.8 \%)$

$125(2.7 \%)$

$83(3.1 \%)$

$194(4.3 \%)$

$100(3.7 \%)$

$0.1 \pm 0.4(0.0)$

$1.5 \pm 1.0(1.0)$

$\begin{array}{ll}2761(60.5 \%) & 1746(64.9 \%) \\ 1803(39.5 \%) & 946(35.1 \%)\end{array}$


Patients with NSCLC identified by ICD-O-3 site recode among all patients with lung cancer (cancer site of C34.0-C34.9) in SEER medicare registry

139,108 patients

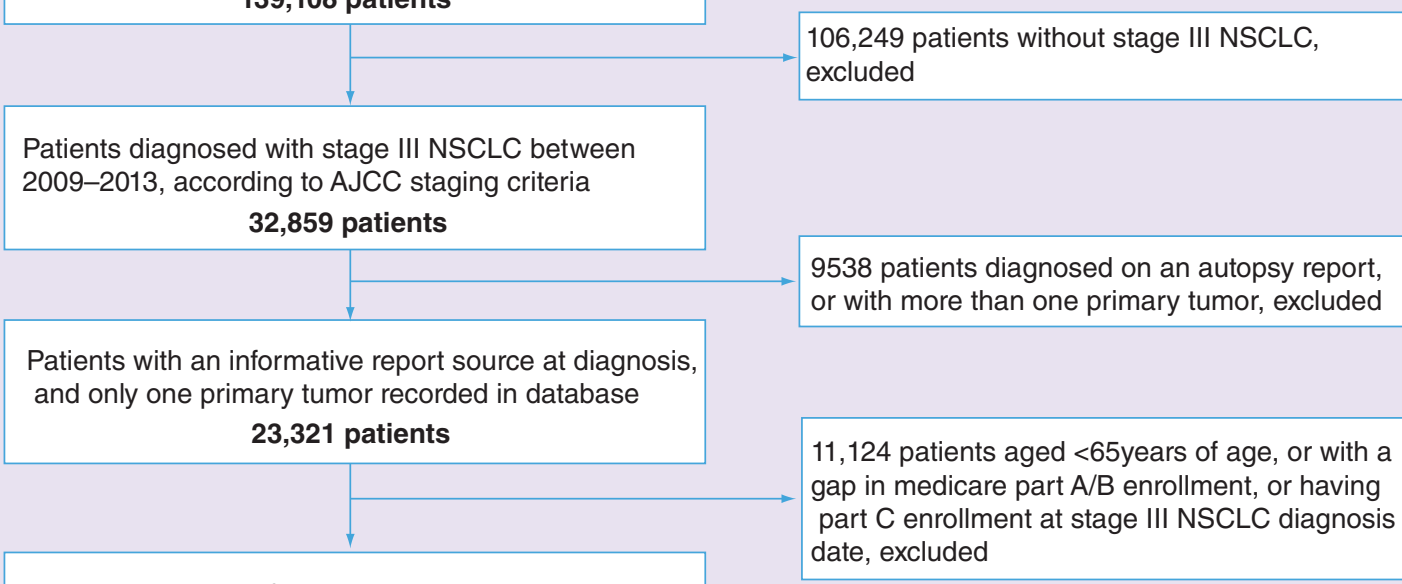

Patients $\geq 65$ years of age with continuous medicare part $\mathrm{A} / \mathrm{B}$ enrollment and no part $\mathrm{C}(\mathrm{HMO})$ enrollment at SEER diagnosis date

12,197 patients

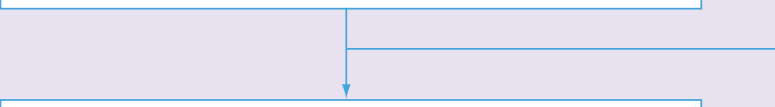

1780 patients with surgical resection of tumor excluded

Patients without surgical resection of tumor performed within 4 months after the diagnosis date

10,417 patients

\begin{tabular}{|l|}
\hline $\begin{array}{l}\text { Patients with } \geq 1 \text { claim for any NSCLC therapy } 30 \text { days } \\
\text { prior to or } 90 \text { days after NSCLC diagnosis date. The } \\
\text { date of initiation of the first therapy will be defined as } \\
\text { the index date }\end{array}$ \\
\hline $\begin{array}{l}\text { Patients with continuous insurance eligibility } \geq 6 \\
\text { months prior to, and } \geq 1 \text { month following, the initiation } \\
\text { of first therapy } \\
4564 \text { patients }\end{array}$ \\
\hline
\end{tabular}

4812 patients who did not initiate NSCLC systemic therapy, excluded
1041 patients without continuous medicare eligibility around date of initiation of first therapy for stage III NSCLC, excluded

Figure 1. Sample selection of patients with unresected, stage III non-small-cell lung cancer.

AJCC: American Joint Committee on Cancer; ICD-O-3: International Classification of Disease for Oncology-3; NSCLC: Non-small cell lung carcinoma; SEER: Surveillance, Epidemiology, and End Results.

Among patients treated with chemotherapy (with or without radiation), the most frequent regimens were carboplatin + paclitaxel (53.6\%), carboplatin + pemetrexed (10.6\%) and cisplatin + etoposide (8.5\%). Patients treated with chemotherapy, who did not receive maintenance therapy $(97 \%)$, had a shorter average duration of treatment compared with those who did (3\%; 96.3 vs 286.2 days). Approximately half of patients treated with chemotherapy $(43.3 \%)$ died during their first therapeutic regimen period. Among patients treated with CRT, the most common chemotherapy regimens were carboplatin + paclitaxel $(62.6 \%)$ and cisplatin + etoposide (11.3\%; Table 3). The mean chemotherapy duration for patients treated with CRT was 89.0 days, and $40.8 \%$ of patients 


\begin{tabular}{|c|c|c|}
\hline First therapeutic regimen & n (\%) & $\begin{array}{l}\text { Duration of regimen } \\
\text { mean } \pm S D \text { (median) }\end{array}$ \\
\hline Carboplatin + paclitaxel & $2050(53.6 \%)$ & $93.0 \pm 54.5(77.0)$ \\
\hline Carboplatin + pemetrexed & $406(10.6 \%)$ & $149.9 \pm 144.1(105.0)$ \\
\hline Cisplatin + etoposide & $327(8.5 \%)$ & $73.4 \pm 37.6(63.0)$ \\
\hline Carboplatin + gemcitabine & $153(4.0 \%)$ & $98.8 \pm 55.4(92.0)$ \\
\hline Carboplatin + docetaxel & $135(3.5 \%)$ & $90.1 \pm 52.3(75.0)$ \\
\hline Chemotherapy with maintenance therapies & $149(3.3 \%)$ & $286.2 \pm 213.7(206.0)$ \\
\hline Carboplatin + paclitaxel + pemetrexed & $56(37.6 \%)$ & $236.2 \pm 166.0(194.5)$ \\
\hline Carboplatin + paclitaxel + erlotinib & $16(10.7 \%)$ & $370.3 \pm 258.0(328.5)$ \\
\hline Carboplatin + pemetrexed + erlotinib & $15(10.1 \%)$ & $374.2 \pm 280.4(282.0)$ \\
\hline Claim for a new agent not in regimen & $1502(37.8 \%)$ & $99.9 \pm 69.7(77.0)$ \\
\hline Gap of more than 90 days & $660(16.6 \%)$ & $119.4 \pm 102.2(94.0)$ \\
\hline Death & $1721(43.3 \%)$ & $84.5 \pm 60.9(70.0)$ \\
\hline Targeted therapy & $219(4.8 \%)$ & $269.3 \pm 293.5(161.0)$ \\
\hline Erlotinib & $181(82.6 \%)$ & $281.3 \pm 306.0(173.0)$ \\
\hline \multicolumn{3}{|l|}{ Targeted therapy classification of discontinuation } \\
\hline Claim for a new agent not in regimen & $57(25.1 \%)$ & $253.4 \pm 198.0(219.0)$ \\
\hline Gap of more than 90 days & $26(11.5 \%)$ & $340.6 \pm 352.4(207.0)$ \\
\hline Death & $127(55.9 \%)$ & $249.0 \pm 310.8(110.5)$ \\
\hline Bevacizumab & $327(7.2 \%)$ & $166.2 \pm 144.6(133.0)$ \\
\hline Bevacizumab + carboplatin + pemetrexed & $118(36.1 \%)$ & $213.7 \pm 142.3(175.5)$ \\
\hline \multicolumn{3}{|l|}{ Bevacizumab classification of discontinuation } \\
\hline Claim for a new agent not in regimen & $163(45.0 \%)$ & $174.8 \pm 138.8(133.0)$ \\
\hline Gap of more than 90 days & $47(13.0 \%)$ & $252.0 \pm 186.2(195.5)$ \\
\hline Death & $148(40.9 \%)$ & $130.3 \pm 122.0(101.5)$ \\
\hline
\end{tabular}

died during their first therapeutic regimen. Among the subgroup of patients treated with CRT, $37.4 \%(\mathrm{n}=1008)$ initiated treatment in 2009-2010, 40.8\% ( $\mathrm{n}=1099)$ initiated treatment in 2011-2012, 21.7\% ( $\mathrm{n}=585)$ initiated treatment in 2013-2014.

Among patients who initiated therapy with a bevacizumab-containing regimen, the most frequently observed regimens were bevacizumab + carboplatin + pemetrexed $(36.1 \%)$ and bevacizumab + carboplatin + paclitaxel (34.6\%; Table 2). The mean duration of treatment for patients treated with a bevacizumab-containing regimen was 166.2 days, and $40.9 \%$ died during their first therapeutic regimen period. Among the 219 patients who received targeted therapy as part of their first therapeutic regimen, the majority were treated with erlotinib $(82.6 \%)$, with a mean duration of therapy of 269.3 days. Among patients with unresected, stage III receiving targeted therapy, $55.9 \%$ died while on their first therapy.

Over a third $(\mathrm{n}=1722,37.0 \%)$ of the study population received a second therapy; of these, $75.5 \%$ received chemotherapy as their second therapeutic regimen (Table 4). The most frequently received second chemotherapy regimens were docetaxel (15.0\%), carboplatin + paclitaxel $(14.2 \%)$ and pemetrexed (12.2\%). For those patients receiving chemotherapy as their first therapy, the duration of second therapy was not longer than for first therapy (111.1 and 105.5 days, respectively). Among the 277 patients with unresected, stage III NSCLC who received targeted therapy as their first therapy, $25.1 \%$ received a second therapy. These patients had a mean (SD) duration of treatment of 154.5 days for their second therapy regimen. 


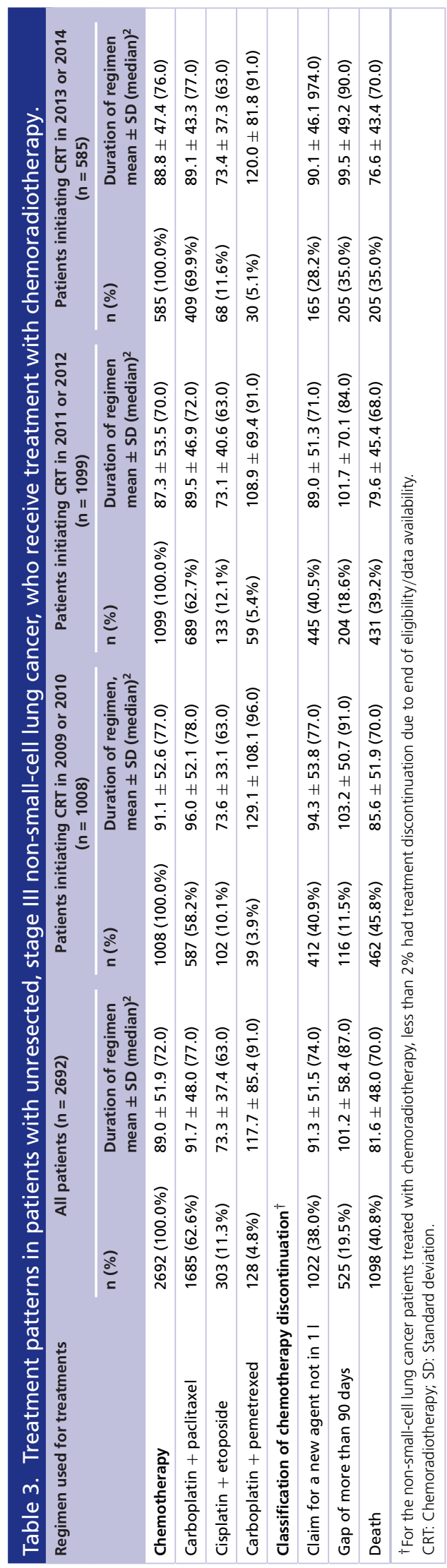


Table 4. Second therapeutic regimen treatment patterns in patients with unresected, stage III non-small-cell

\section{lung cancer.}

\begin{tabular}{|c|c|c|}
\hline Regimen used for treatments & n (\%) & $\begin{array}{l}\text { Duration of regimen } \\
\text { mean } \pm S D \text { (median) }\end{array}$ \\
\hline Chemotherapy & $1300(75.5 \%)$ & $96.2 \pm 96.7(70.0)$ \\
\hline Docetaxel & $195(15.0 \%)$ & $79.4 \pm 68.9(64.0)$ \\
\hline Carboplatin + paclitaxel & $184(14.2 \%)$ & $84.6 \pm 70.4(63.0)$ \\
\hline Pemetrexed & $159(12.2 \%)$ & $136.3 \pm 155.8(91.0)$ \\
\hline Gemcitabine & $155(11.9 \%)$ & $99.0 \pm 91.5(70.0)$ \\
\hline Carboplatin + gemcitabine & $134(10.3 \%)$ & $84.0 \pm 52.5(71.0)$ \\
\hline \multicolumn{3}{|l|}{ Chemotherapy classification of discontinuation } \\
\hline Claim for a new agent not in regimen & $521(40.1 \%)$ & $100.6 \pm 80.1(71.0)$ \\
\hline Gap of more than 90 days & $133(10.2 \%)$ & $106.0 \pm 111.6(70.0)$ \\
\hline Death & $581(44.7 \%)$ & $84.4 \pm 93.2(62.0)$ \\
\hline Targeted therapy & $163(9.5 \%)$ & $185.0 \pm 255.3(91.0)$ \\
\hline Erlotinib & $121(74.2 \%)$ & $203.8 \pm 286.9(91.0)$ \\
\hline \multicolumn{3}{|c|}{ Targeted therapy classification of discontinuation } \\
\hline Claim for a new agent not in regimen & $43(26.4 \%)$ & $184.8 \pm 181.8(132.0)$ \\
\hline Gap of more than 90 days & $11(6.1 \%)$ & $163.9 \pm 137.9(103.5)$ \\
\hline Death & $88(54.0 \%)$ & $119.2 \pm 194.8(61.0)$ \\
\hline Bevacizumab & $259(15.0 \%)$ & $151.2 \pm 143.4(104.0)$ \\
\hline Bevacizumab + carboplatin + pemetrexed & $63(24.3 \%)$ & $194.9 \pm 180.4(147.0)$ \\
\hline Bevacizumab + carboplatin + paclitaxel & $61(23.6 \%)$ & $128.0 \pm 123.6(90.0)$ \\
\hline Bevacizumab + pemetrexed & $53(20.5 \%)$ & $159.2 \pm 133.9(111.0)$ \\
\hline \multicolumn{3}{|l|}{ Bevacizumab classification of discontinuation } \\
\hline Claim for a new agent not in regimen & $116(44.8 \%)$ & $155.0 \pm 128.8(119.0)$ \\
\hline Gap of more than 90 days & $43(16.6 \%)$ & $213.3 \pm 163.7(189.0)$ \\
\hline Death & $91(35.1 \%)$ & $126.3 \pm 147.2(76.0)$ \\
\hline \multicolumn{3}{|c|}{$\begin{array}{l}\text { All patients, } n=1722 \text {. } \\
\text { For the non-small-cell lung cancer patients treated with a second therapeutic regimen, less than } 10 \% \text { had treatment discontinuation due to end of eligibility/data availability. } \\
\text { SD: Standard deviation. }\end{array}$} \\
\hline
\end{tabular}

\section{Overall survival}

Among the total population of patients with unresected, stage III NSCLC who initiated treatment with chemotherapy, the median (interquartile range) OS was $13.2(6.0,28.9)$ months (Figure 2). Among the subgroup of these patients who initiated treatment with CRT, the median OS was $14.8(6.7,33.4)$ months (Figure 3). The proportion of patients alive at 1 year following the index date was 56\% for patients initiating CRT in 2009-2010, and 58\% for patients initiating CRT in either 2011-2012 or 2013-2014; survival rates did not differ upon adjustment for differences in baseline characteristics, such as patient age, sex, race, region, histology, disease grade, laterality, tumor site, number and location of metastatic sites, CCI and predicted PS, between the index year groups $(\mathrm{p}=0.827)$.

\section{Discussion}

This study aimed to understand real-world treatment patterns and clinical outcomes for patients diagnosed with unresected, stage III NSCLC between 2009 to 2013, prior to recent developments of novel therapeutic strategies for NSCLC. Among patients who were treated with a systemic therapy, 59\% received treatment with CRT, which is consistent with national guidelines recommending CRT in this group of patients [7]. Most patients initiated treatment with a platinum-chemotherapy doublet regimen; the most frequently observed chemotherapy regimens were carboplatin + paclitaxel and carboplatin + pemetrexed. A small proportion of patients had regimens containing bevacizumab or targeted therapy agents, with or without chemotherapy.

Concurrent CRT remains the standard-of-care treatment for unresectable, stage III NSCLC patients with platinum-doublet chemotherapy combined with radiation as the most commonly administered regimen [6-8]. There are limited real-world studies on trends of CRT among these patients. Consistent with our findings, a previous analysis conducted on patients treated within the Veterans Health Administration found that, among patients 


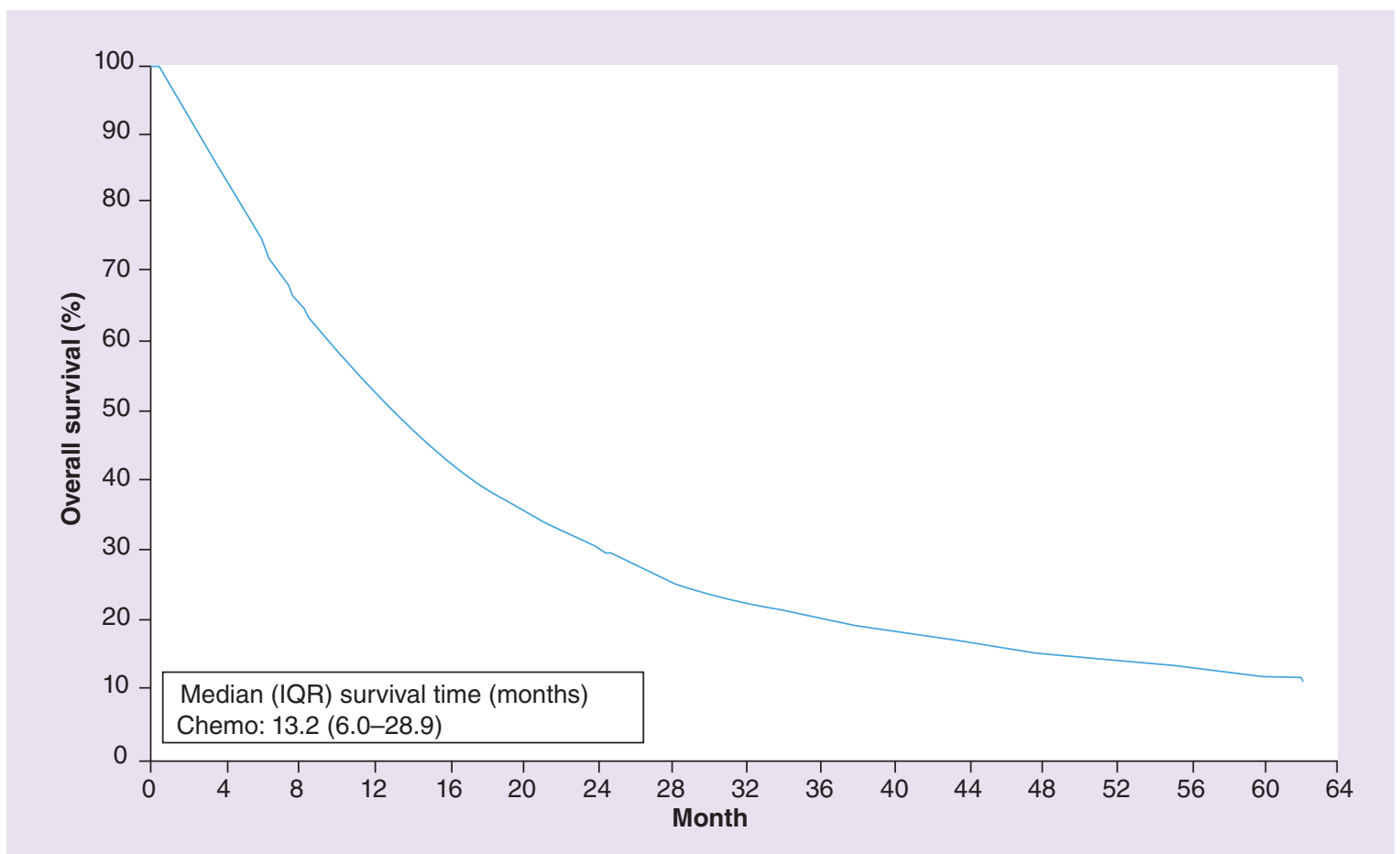

Figure 2. Kaplan-Meier curve for overall survival in patients with unresected, stage III non-small-cell lung cancer, who receive treatment with chemotherapy.

IQR: Inter quartile range.

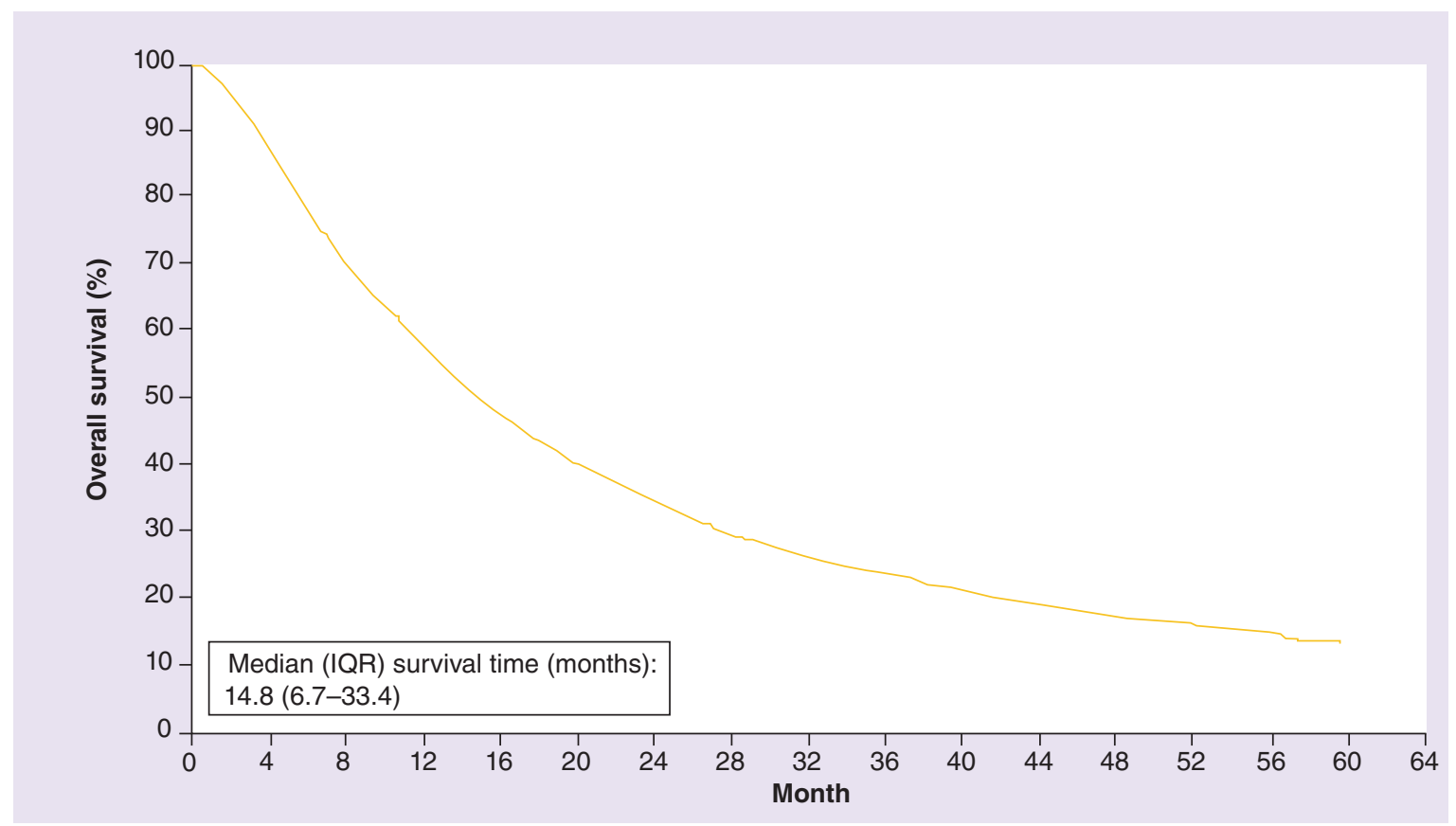

Figure 3. Kaplan-Meier curve for overall survival in patients with unresected, stage III non-small-cell lung cancer, who receive treatment with chemoradiotherapy.

IQR: Inter quartile range. 
treated with concurrent CRT between 2001 and 2010, carboplatin + paclitaxel was used to treat approximately three-quarters of patients while cisplatin + etoposide was used in a quarter [17].

Chemotherapy, given with or without concurrent radiotherapy, plays a critical role in the management of unresectable, stage III NSCLC; the combined modality treatment has been shown to improve survival over chemotherapy alone [18-20]. In this study, median OS over the entire treatment period was 13.2 months for patients who initiated chemotherapy (with or without radiation) and 14.8 months for the subgroup of patients who initiated CRT. These findings are consistent with previous real-world studies that have analyzed survival among unresectable, stage III NSCLC patients, which have generated estimates of median OS ranging from 14 to 19 months [6,17,21,22]. Recent randomized trials have shown a longer median OS of approximately 24 months in unresectable, stage III NSCLC patients treated with concurrent CRT [12]. However, patients participating in trials are generally younger and have fewer co-morbidities than the SEER-Medicare study population.

This study found that OS did not improve over time among the subgroup of patients who received treatment with CRT from 2009 to 2014. This stagnancy in clinical outcomes reflects the lack of more effective therapy options and limited improvements in CRT for these patients over this time period. Since the advent of CRT in the 1990s, great efforts have been made to further improve treatment of stage III NSCLC, however, they have been unsuccessful [23]. Newer therapies such as tyrosine kinase inhibitors and immunotherapies have modified the stage IV NSCLC treatment landscape; however, until the recent approval of durvalumab, there were no major therapeutic advances in treatment options for stage III patients [13,24]. Durvalumab is a selective, high-affinity, engineered human IgG1 monoclonal antibody that blocks the immunosuppressive effect of PD-L1 binding to PD-1 receptors and CD80, thereby allowing T cells to recognize and kill tumor cells [25]. Published results from the randomized, double-blind, Phase III PACIFIC trial demonstrated that treatment with durvalumab in patients who did not have disease progression after two or more cycles of platinum-based concurrent CRT significantly increased progression-free survival from 5.6 to 16.8 months, while maintaining a safety profile comparable to placebo [26]. Recently, published results for OS from the PACIFIC trial indicate that durvalumab significantly prolongs survival as compared with placebo, with a 24-month OS rate of $66 \%$ in the treatment group compared with $56 \%$ in the placebo group (hazard ratio for death of $0.68 ; p=0.0025$ ) [27]. Potential treatments such as cytotoxic T-lymphocyte-associated protein- 4 inhibitors, PD-1 and PD-L1 inhibitors, cancer vaccines, and combinations of immuno-oncology with CRT and tyrosine kinase inhibitors are also under investigation in stage III NSCLC, although still in early development [23,28-30]. Immunotherapies are a promising new treatment modality for stage III NSCLC, and the impact of these new therapies on real-world patient outcomes warrants further research.

The use of the SEER-Medicare database for this analysis allows for the evaluation of tumor characteristics and treatment patterns of patients with unresected, stage III NSCLC in a real-world setting. It is a nationally representative database, thus, results from this study are more generalizable compared with studies limited to claims data from specific states. In addition, the SEER-Medicare database includes a validated date of death, which allows for a reliable and accurate evaluation of OS. The use of the SEER cancer registries for diagnosis data ameliorates misclassification of NSCLC from use of International Classification of Diseases, Ninth Revision, Clinical Modification (ICD-9-CM) codes only, as in common in real-world data sources only comprised of claims data. This study went further than previous SEER-Medicare-based analyses of NSCLC patients by assessing predicted PS, an important confounder not otherwise available in claims data, using a previously validated claims-based algorithm, as well as assessing use of maintenance therapy.

Although this was a robust analysis, the results should be viewed in light of certain limitations. First, the SEERMedicare-linked database only includes data for those aged 65 years and older and may not capture all patients with NSCLC. Therefore, the study results are only generalizable to Medicare-eligible patients and may not be applicable to other populations, such as younger patients enrolled in a commercial insurance plan. Second, due to the way that radiotherapy is currently identified in the SEER data, it is not possible to identify salvage radiotherapy, nor distinguish between sequential versus concurrent CRT using SEER data alone. In future analyses, Medicare claims may be helpful in distinguishing between these therapies. This distinction is important, as previous studies have shown that patients receiving concurrent CRT have improved survival outcomes compared with those receiving sequential CRT [9]. Third, previous studies have shown some degree of discrepancy between receipt of radiation as recorded SEER data and Medicare claims [31,32], and there could be similar issues in the present study. Last, SEER-Medicare data are released with a lag of several years, limiting the ability of the analysis to extend beyond 2014 at the time of conduct; however, the treatment options for stage III disease have remained largely unchanged, making our results relevant and informative. 


\section{Conclusion}

Treatment patterns and survival outcomes in patients with unresected, stage III NSCLC have remained static across years, indicating a lack of improvement in outcomes for patients from 2009 to 2014. The results of this study indicate an unmet need for more effective therapy options for these patients.

\section{Summary points}

- The standard-of-care for patients with unresectable, stage III non-small-cell lung cancer (NSCLC) during the study period (2009-2014) was chemoradiotherapy (CRT), followed by active surveillance.

- This retrospective longitudinal cohort study assessed treatment patterns and compared 1-year overall survival (OS) across 2009-2014 among patients aged $\geq 65$ years with unresected, stage III NSCLC, using Surveillance, Epidemiology, and End Results-Medicare data. Patients were required to have $\geq 1$ claim for an NSCLC therapy within 90 days of diagnosis (index date), and no surgical resection of tumor.

- Of the 4564 study patients, $84 \%$ of patients initiated chemotherapy (with or without radiotherapy), and only $59 \%$ of patients initiated CRT as their first therapeutic regimen.

- During the study period, median OS among patients treated with chemotherapy was 13.2 (interquartile range: 6.0-28.9) months, and among patients treated with CRT was 14.8 (6.7-33.4) months.

- CRT patients were further divided into cohorts defined by index year of CRT initiation (i.e., patients who initiated CRT in 2009-2010, 2011-2012 and 2013-2014). 1-year OS was 56\% in patients treated with CRT who initiated treatment in 2009-2010, 58\% in 2011-2012 and 58\% in 2013-2014 and there was no difference across index years after adjustment for baseline covariates ( $p=0.827$ ).

- Therapies such as tyrosine kinase inhibitors and immunotherapies have modified the treatment landscape of stage IV NSCLC, but there were no major therapeutic advances in treatment options for stage III NSCLC patients until the February 2018 approval of durvalumab, a selective, high-affinity, engineered monoclonal antibody that has shown promising efficacy and safety results for patients with stage III NSCLC whose disease had not progressed following concurrent platinum-based CRT.

- This retrospective study adds to the literature because it aimed to understand real-world treatment patterns and clinical outcomes of patients with stage III, unresectable NSCLC. Results demonstrate stagnation in clinical outcomes in stage III NSCLC patients during the study period, thus reflecting an unmet need for effective therapeutic options in this patient population.

\section{Author contributions}

All the authors conceived and designed the study. A Dua, P Bobbili and MS Duh collected and assembled the data. All the authors contributed to the data analysis, interpretation, manuscript writing and approved the final version of the manuscript.

\section{Acknowledgments}

This study used the linked SEER-Medicare database. The interpretation and reporting of these data are the sole responsibility of the authors. The authors acknowledge the efforts of the National Cancer Institute; the Office of Research, Development and Information, CMS; Information Management Services (IMS), Inc.; and the Surveillance, Epidemiology, and End Results (SEER) Program tumor registries in the creation of the SEER-Medicare database.

\section{Financial \& competing interests disclosure}

This work was supported by AstraZeneca. P Bobbili, A Dua and MS Duh are employees of Analysis Group Inc., a consulting company that have received research funds from AstraZeneca to conduct this study. K Ryan, AW Fernandes and M Pavilack are employees of AstraZeneca and own stock in AstraZeneca. JE Gomez is an employee of Mount Sinai Hospital, and has received consultancy fees and research support from AstraZeneca. The authors have no other relevant affiliations or financial involvement with any organization or entity with a financial interest in or financial conflict with the subject matter or materials discussed in the manuscript apart from those disclosed.

Medical writing support was provided by Analysis Group and was funded by AstraZeneca.

Ethical conduct of research

The authors state that they have obtained appropriate institutional review board approval. Informed consent from study participants was not required as this was a retrospective analysis of an existing database. 
Open access

This work is licensed under the Attribution-NonCommercial-NoDerivatives 4.0 Unported License. To view a copy of this license, visit http://creativecommons.org/licenses/by-nc-nd/4.0/

\section{References}

Papers of special note have been highlighted as: $\bullet \bullet$ of considerable interest

1. American Cancer Society. Cancer Facts and Figures 2018 (2018). www.cancer.org/content/dam/cancer-org/research/cancer-facts-and-st atistics/annual-cancer-facts-and-figures/2018/cancer-facts-and-figures-2018.pdf

2. American Cancer Society. Non-small cell lung cancer survival rates, by stage (2017). www.cancer.org/cancer/non-small-cell-lung-cancer/detection-diagnosis-staging/survival-rates.html

3. American Cancer Society. Lung cancer (non-small cell) overview (2018). www.cancer.org/cancer/non-small-cell-lung-cancer/about/what-is-non-small-cell-lung-cancer.html

4. Wauters E, Vansteenkiste J. Unresectable stage III non-small cell lung cancer: is tecemotide a new START for our patients? J. Thorac. Dis. 6(6), 574-577 (2014).

5. Ali A, Goffin JR, Arnold A, Ellis PM. Survival of patients with non-small-cell lung cancer after a diagnosis of brain metastases. Curr. Oncol. 20(4), e300-e306 (2013).

6. Yoon SM, Shaikh T, Hallman M. Therapeutic management options for stage III non-small cell lung cancer. World J. Clin. Oncol. 8(1), 1-20 (2017).

-• Review of potential treatment options for the management of advanced non-small-cell lung cancer (NSCLC), which outlines concurrent chemoradiotherapy (CRT) as the preferred treatment option for patients with unresectable disease.

7. National Comprehensive Cancer Network (NCCN). NCCN guidelines for patients: lung cancer - non-small cell lung cancer 2018 (2018). www.cancer.org/cancer/non-small-cell-lung-cancer/treating.html

8. Eberhardt WE. Concurrent chemoradiotherapy in stage III non-small-cell lung cancer: what is the best regimen? J. Clin. Oncol. 33(6), 532-533 (2015).

-• Demonstrates the unmet need for patients with stage III NSCLC, and outlines the needs for additional prospective randomized clinical trials in this high-risk population to determine potential treatment options.

9. Auperin A, Le Pechoux C, Rolland E et al. Meta-analysis of concomitant versus sequential radiochemotherapy in locally advanced non-small-cell lung cancer. J. Clin. Oncol. 28(13), 2181-2190 (2010).

-• Demonstrates the benefit of concurrent radiotherapy compared with sequential chemotherapy on overall survival (OS) of patients with advanced NSCLC.

10. Lilenbaum R, Samuels M, Wang X et al. A Phase II study of induction chemotherapy followed by thoracic radiotherapy and erlotinib in poor-risk stage III non-small-cell lung cancer: results of CALGB 30605 (Alliance)/RTOG 0972 (NRG). J. Thorac. Oncol. 10(1), 143-147 (2015).

11. Vokes EE, Herndon JE 2nd, Kelley MJ et al. Induction chemotherapy followed by chemoradiotherapy compared with chemoradiotherapy alone for regionally advanced unresectable stage III non-small-cell lung cancer: cancer and leukemia group B. J. Clin. Oncol. 25(13), 1698-1704 (2007).

12. Bradley JD, Paulus R, Komaki R et al. Standard-dose versus high-dose conformal radiotherapy with concurrent and consolidation carboplatin plus paclitaxel with or without cetuximab for patients with stage IIIA or IIIB non-small-cell lung cancer (RTOG 0617): a randomised, two-by-two factorial Phase III study. Lancet Oncol. 16(2), 187-199 (2015).

13. Mezquita L, Planchard D. Durvalumab for the treatment of non-small cell lung cancer. Expert Rev. Respir. Med. 12(8), 627-639 (2018).

14. Warren JL, Klabunde CN, Schrag D, Bach PB, Riley GF. Overview of the SEER-Medicare data: content, research applications, and generalizability to the United States elderly population. Med. Care 40(Suppl. 8), IV-3-18 (2002).

15. Salloum RG, Smith TJ, Jensen GA, Lafata JE. Using claims-based measures to predict performance status score in patients with lung cancer. Cancer 117(5), 1038-1048 (2011).

16. Lang K, Marciniak MD, Faries D et al. Trends and predictors of first-line chemotherapy use among elderly patients with advanced non-small cell lung cancer in the United States. Lung Cancer 63(2), 264-270 (2009).

17. Santana-Davila R, Devisetty K, Szabo A et al. Cisplatin and etoposide versus carboplatin and paclitaxel with concurrent radiotherapy for stage III non-small-cell lung cancer: an analysis of Veterans Health Administration data. J. Clin. Oncol. 33(6), 567-574 (2015).

18. Curran WJ Jr, Paulus R, Langer CJ et al. Sequential vs. concurrent chemoradiation for stage III non-small cell lung cancer: randomized Phase III trial RTOG 9410. J. Natl. Cancer Inst. 103(19), 1452-1460 (2011).

-• Phase III study demonstrating that concurrent cisplatin-based chemotherapy and thoracic radiotherapy is associated with better survival outcomes compared with sequential delivery of the same treatment combination.

19. Pritchard RS, Anthony SP. Chemotherapy plus radiotherapy compared with radiotherapy alone in the treatment of locally advanced, unresectable, non-small-cell lung cancer. A meta-analysis. Ann. Intern. Med. 125(9), 723-729 (1996). 
20. Non-small Cell Lung Cancer Collaborative Group. Chemotherapy in non-small cell lung cancer: a meta-analysis using updated data on individual patients from 52 randomised clinical trials. BMJ311(7010), 899-909 (1995).

21. Urvay SE, Yucel B, Erdis E, Turan N. Prognostic factors in stage III non-small-cell lung cancer patients. Asian Pac. J. Cancer Prev. 17(10), 4693-4697 (2016).

22. Harris JP, Patel MI, Loo BW, Wakelee HA, Diehn M. A population-based comparative effectiveness study of chemoradiation regimens and sequences in stage III non-small cell lung cancer. Lung Cancer 108, 173-182 (2017).

-. Population-based study utilizing SEER-Medicare data that demonstrated improved OS for patients receiving consolidation chemotherapy with carboplatin, and comparable OS for patients receiving consolidation therapy with cisplatin.

23. Kumar SS, Higgins KA, McGarry RC. Emerging therapies for stage III non-small cell lung cancer: stereotactic body radiation therapy and immunotherapy. Front. Oncol. 7, 197 (2017).

24. McCall NS, Dicker AP, Lu B. Beyond concurrent chemoradiation: the emerging role of PD-1/PD-L1 inhibitors in stage III lung cancer. Clin. Cancer Res. 24(6), 1271-1276 (2018).

-• Review of incorporating PD1/PDL1 inhibitors in treatment of patients with unresectable stage III NSCLC following interim results of the PACIFIC trial demonstrating significant improvement in median progression-free survival with consolidation postconcurrent CRT.

25. Stewart R, Morrow M, Hammond SA et al. Identification and characterization of MEDI4736, an antagonistic anti-PD-L1 monoclonal antibody. Cancer Immunol. Res. 3(9), 1052-1062 (2015).

26. Antonia SJ, Villegas A, Daniel D et al. Durvalumab after chemoradiotherapy in stage III non-small-cell lung cancer. N. Engl. J. Med. 377(20), 1919-1929 (2017).

-• Phase III study demonstrating significantly higher progression-free survival in patients treated with durvalumab versus placebo in patients with stage III NSCLC without disease progression after platinum-based CRT.

27. Antonia SJ, Villegas A, Daniel D et al. Overall survival with durvalumab after chemoradiotherapy in stage III NSCLC. N. Engl. J. Med. 379(24), 2342-2350 (2018).

28. Schild SE, Vokes EE. Pathways to improving combined modality therapy for stage III non-small-cell lung cancer. Ann. Oncol. 27(4), 590-599 (2016).

29. Moya-Horno I, Viteri S, Karachaliou N, Rosell R. Combination of immunotherapy with targeted therapies in advanced non-small cell lung cancer (NSCLC). Ther. Adv. Med. Oncol. 10, 1758834017745012 (2018).

30. Somasundaram A, Burns TF. The next generation of immunotherapy: keeping lung cancer in check. J. Hematol. Oncol. 10(1), 87 (2017).

31. Virnig BA, Warren JL, Cooper GS, Klabunde CN, Schussler N, Freeman J. Studying radiation therapy using SEER-Medicare-linked data. Med. Care 40(Suppl. 8), IV-49-54 (2002).

32. Noone A-M, Lund JL, Mariotto A et al. Comparison of SEER treatment data with Medicare claims. Med. Care 54(9), e55-e64 (2016). 
